\title{
An Efficient Method to Find Solutions for Transcendental Equations with Several Roots
}

\author{
Rogelio Luck, ${ }^{1}$ Gregory J. Zdaniuk, ${ }^{2}$ and Heejin Cho ${ }^{1}$ \\ ${ }^{1}$ Department of Mechanical Engineering, Mississippi State University, P.O. Box 9552, Mississippi State, MS 39762, USA \\ ${ }^{2}$ Cofely UK, Kings Yard, 1 Waterden Road, Queen Elizabeth Olympic Park, London E15 2GP, UK \\ Correspondence should be addressed to Heejin Cho; cho@me.msstate.edu
}

Received 17 July 2015; Revised 6 October 2015; Accepted 7 October 2015

Academic Editor: Song Cen

Copyright (C) 2015 Rogelio Luck et al. This is an open access article distributed under the Creative Commons Attribution License, which permits unrestricted use, distribution, and reproduction in any medium, provided the original work is properly cited.

\begin{abstract}
This paper presents a method for obtaining a solution for all the roots of a transcendental equation within a bounded region by finding a polynomial equation with the same roots as the transcendental equation. The proposed method is developed using Cauchy's integral theorem for complex variables and transforms the problem of finding the roots of a transcendental equation into an equivalent problem of finding roots of a polynomial equation with exactly the same roots. The interesting result is that the coefficients of the polynomial form a vector which lies in the null space of a Hankel matrix made up of the Fourier series coefficients of the inverse of the original transcendental equation. Then the explicit solution can be readily obtained using the complex fast Fourier transform. To conclude, the authors present an example by solving for the first three eigenvalues of the 1D transient heat conduction problem.
\end{abstract}

\section{Introduction}

The determination of roots of transcendental functions is a problem commonly encountered in a broad spectrum of engineering applications, such as heat transfer (e.g., [14]), dynamics and control (e.g., [5]), sound and vibration (e.g., $[6,7]$ ), and quantum mechanics (e.g., [8]). There exist a wide variety of numerical methods that are useful for approximating the solution to any desired degree of accuracy. General descriptions of common root finding techniques are available in most textbooks in the area of numerical analysis, for example, Chapra and Canale [9].

Explicit methods have also been developed to find roots of transcendental equations. Explicit solutions can provide more insight into the problem under consideration. They are also useful, for example, in the development of analytical derivatives for uncertainty analyses and sensitivity studies or for checking convergence of approximate root finding techniques [3]. Leathers and McCormick [10] addressed a methodology for obtaining explicit solutions to several transcendental problems arising in heat transfer. The general approach used was based on methods of Muskhelishvili [11] and was developed by Burniston and Siewert [12]. This approach "depends on formulating an appropriate Riemann problem of complex variable theory and then expressing the solution of the transcendental equation in terms of a canonical solution of that problem" [10]. Luck and Stevens [13] formulated an explicit expression for a single root of any analytic transcendental function. Their method is based on Cauchy's integral theorem and uses only basic concepts of complex integration to determine the root of a function by locating the singularity of the reciprocal of the function. It has now been found that this idea was initially proposed by Jackson $[14,15]$. The current paper extends their idea presenting a method for finding several roots of transcendental equations within a bounded region by transforming the problem into an equivalent problem of finding roots of a polynomial equation with exactly the same roots. It follows that an explicit solution is readily available through the complex fast Fourier transform (cfft) when dealing with up to four roots at a time. For five or more roots the solution is implicit according to the Abel-Ruffini theorem [16]. The implicitness of finding roots of fifth- and higher-order polynomials is not really an issue as algorithms 
designed for this task are readily available (McNamee [17-20], McNamee and Pan [21]) or larger problems can be divided into smaller problems by analyzing nonoverlapping bounded regions with four roots or less in each region.

\section{Method Development}

Suppose $f(x)$ is a transcendental function with $N$ roots within an interval of width $2 \cdot R$ and center $h$ such that $h-R<$ $x<h+R$. Let $p(x)$ be an $N$ th-order polynomial with roots located at exactly the same locations as the roots of $f(x)$ in the interval $h-R<x<h+R$. The ratio $p(x) / f(x)$ is an analytic function in the interval $h-R<x<h+R$ because the poles of $1 / f(x)$ in the interval are cancelled out by the roots of $p(x)$. The next step in the analysis is to normalize the variable of interest. Let

$$
y=\frac{x-h}{R}
$$

such that $-1<y<1$. Functions $f(x)$ and $p(x)$ can be recast as

$$
\begin{aligned}
& f(x)=f(R \cdot y+h)=F(y), \\
& p(x)=p(R \cdot y+h)=P(y),
\end{aligned}
$$

where

$$
P(y)=\sum_{k=0}^{N}\left(c_{k} y^{k}\right)
$$

and $N$ is the number of roots in the interval.

Now consider the function $P(y) / F(y)$ in the interval $-1<$ $y<1$. If the analysis is extended into the complex plane (by substituting the real variable $y$ with the complex variable $z$ ), integration around a circular path of radius 1 yields

$$
\oint \frac{P(z)}{F(z)} d z=0
$$

which is a consequence of Cauchy's theorem regarding the line integral of a function that is analytic on and inside of the contour defined by the closed path of integration.

Equation (4) can be used to find the coefficients $c_{k}$ of polynomial $P(y)$ as follows:

$$
\oint \frac{P(z)}{F(z)} d z=\oint \frac{\sum_{k=0}^{N}\left(c_{k} z^{k}\right)}{F(z)} d z=\sum_{k=0}^{N}\left(c_{k} \oint \frac{z^{k}}{F(z)} d z\right)=0 .
$$

Note that, given a positive integer $r$, it is also true that

$$
\oint \frac{z^{r} P(z)}{F(z)} d z=0
$$

because the function being integrated remains analytic when multiplied by $z^{r}$. Following the approach presented in (5)

$$
\oint \frac{z^{r} P(z)}{F(z)} d z=\sum_{k=0}^{N}\left(c_{k} \oint \frac{z^{k+r}}{F(z)} d z\right)=0 .
$$

Following is the procedure for obtaining coefficients $c_{k}$. First, the integrals are evaluated by substituting

$$
\begin{gathered}
z=e^{j \theta}, \\
d z=j e^{j \theta} d \theta .
\end{gathered}
$$

Let

$$
G(\theta)=\frac{1}{F\left(e^{j \theta}\right)}
$$

It follows that

$$
\oint \frac{z^{r+k}}{F(z)} d z=\int_{0}^{2 \pi} G(\theta)\left(e^{j \theta}\right)^{r+k} j e^{j \theta} d \theta=j \int_{0}^{2 \pi} G(\theta) e^{j(r+k+1) \theta} d \theta .
$$

Another simplification can be implemented by defining

$$
G_{k}=\int_{0}^{2 \pi} G(\theta) e^{j k \theta} d \theta .
$$

Note that $G_{k}$ can be interpreted as the $k$ th complex Fourier series coefficient of $G(\theta)$. Finally, substituting $G_{k}$ into (7) and cancelling $j$ yield

$$
\sum_{k=0}^{N}\left(c_{k} G_{r+k+1}\right)=0
$$

Equation (12) is the equation used to find coefficients $c_{0}, c_{1}, c_{2}, \ldots, c_{N}$. Note that (12) is true for any positive integer value of $r$. This means that one can "create" as many equations as there are unknown coefficients $c_{k}$. Varying $r$ from 0 to $N-1$, the following system of equations is obtained:

$$
\left[\begin{array}{cccccc}
G_{1} & G_{2} & G_{3} & G_{4} & \ldots & G_{N+1} \\
G_{2} & G_{3} & G_{4} & G_{5} & \ldots & G_{N+2} \\
G_{3} & G_{4} & G_{5} & G_{6} & \ldots & G_{N+3} \\
\vdots & \vdots & \vdots & \vdots & \ddots & \vdots \\
G_{N} & G_{N+1} & G_{N+2} & G_{N+3} & \ldots & G_{2 N}
\end{array}\right]\left\{\begin{array}{c}
c_{0} \\
c_{1} \\
c_{2} \\
\vdots \\
c_{N}
\end{array}\right\}=0 .
$$

Because (13) represents a system of linearly dependent equations, that is, having fewer equations than unknowns, additional equations are necessary to obtain a solution. One possible equation consists of setting $c_{N}$ equal to any real nonzero number, which allows moving the last column of the $G$ matrix to the right hand side.

Equation (13) is worthy of comment. Matrix $G$ is composed solely of Fourier series coefficients, implying that the equations do not depend on normalization factors used to compute the Fourier series coefficients. In practice, the coefficients of $G$ are approximated through the complex fast Fourier transform (cfft). The cfft approximation is less accurate for the last column of the $G$ matrix because this column corresponds to high-frequency terms. It follows that moving the last column of the $G$ matrix to the right hand side of the equation results in a slightly more robust solution. Furthermore, the system of equations constitutes a Hankel matrix for which an inverse can be easily obtained [22]. Therefore, (13) can be quickly implemented and solved for the coefficients $c_{k}$. Finally, the symmetry or "structure" of (13) leads the authors to believe there is a deeper meaning to this system of equations. 


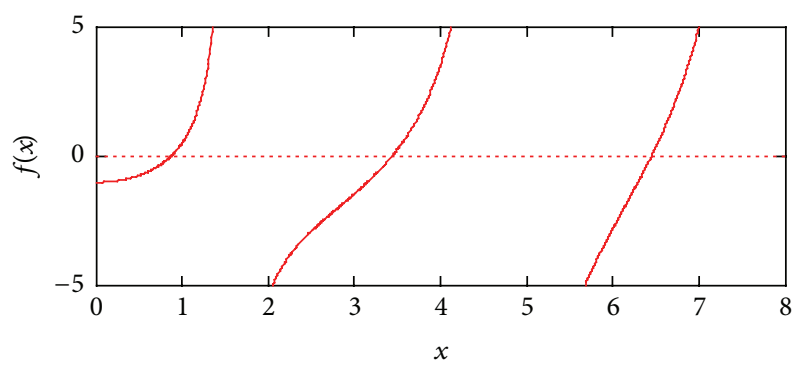

Figure 1: Plot of $f(x)$.

\section{Example}

To illustrate the current method, a simple yet helpful example is described below. The solution of the unsteady heat conduction equation yields the characteristic equation or eigenfunction of the following form [23]:

$$
x \tan x=\mathrm{Bi}
$$

Equation (14) is a transcendental equation with an infinite number of roots called characteristic values or eigenvalues. The characteristic equation in this case is implicit. Arbitrarily assuming $\mathrm{Bi}=1$, (14) can be rewritten as

$$
x \tan x-1=0 .
$$

Let

$$
f(x)=x \tan x-1
$$

The plot of $f(x)$ is shown on Figure 1 .

The method described in the previous section is now applied to solve for the first three positive roots of (16). Figure 1 illustrates that the three roots occur in the interval $0<x<8$, suggesting $h=4, R=4$, and $N=3$. Note that one simple way of approximating coefficients $G_{k}$ is to discretize $G(\theta)$ for $\theta \in[0,2 \pi)$ and to use the complex fast Fourier transform (cfft) readily available in many commercial mathematical software packages (e.g., MATLAB and Mathcad), rather than performing the integral of (11). With $c_{3}=1$ and $N=3$, (13) becomes

$$
\left[\begin{array}{ccc}
G_{1} & G_{2} & G_{3} \\
G_{2} & G_{3} & G_{4} \\
G_{3} & G_{4} & G_{5}
\end{array}\right]\left\{\begin{array}{l}
c_{0} \\
c_{1} \\
c_{2}
\end{array}\right\}=-\left\{\begin{array}{l}
G_{4} \\
G_{5} \\
G_{6}
\end{array}\right\} .
$$

Discretizing $\theta$ into 256 equidistant points, the cfft yields

$$
\left[\begin{array}{ccc}
2.846 & -0.768 & 1.014 \\
-0.768 & 1.014 & -0.476 \\
1.014 & -0.476 & 0.559
\end{array}\right]\left\{\begin{array}{l}
c_{0} \\
c_{1} \\
c_{2}
\end{array}\right\}=\left\{\begin{array}{c}
0.476 \\
-0.559 \\
0.324
\end{array}\right\} .
$$

Solving this system of equations gives

$$
c=\left\{\begin{array}{c}
-0.069 \\
-0.453 \\
0.319 \\
1
\end{array}\right\}
$$

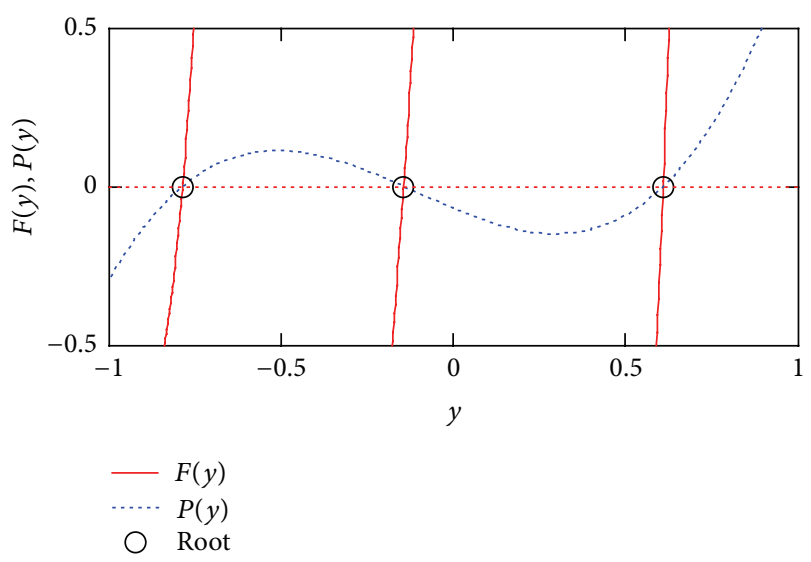

FIgURE 2: Plot of $P(y)$ and $F(y)$ with locations of the roots.

which translates into

$$
P(y)=-0.069-0.453 y+0.319 y^{2}+y^{3} .
$$

Figure 2 plots the original transcendental function $F(y)$ and the polynomial $P(y)$. The plot clearly shows that the polynomial has the same roots as the original function $F(y)$; that is, $y=-0.785, y=-0.144$, and $y=0.609$. The roots of a cubic polynomial can be found using the explicit algebraic expressions given by many authors, for example, Abramowitz and Stegun [24], Press et al. [25], and Weisstein [26]. Finally, undoing the variable normalization yields $x=0.860, x=$ 3.426 , and $x=6.437$ as the roots of $f(x)$ or eigenvalues of the original problem.

\section{Conclusion}

The current paper presents a method for finding simultaneously several roots of a transcendental equation $f(x)$ within a bounded region, by transforming the problem into an equivalent problem of finding roots of a polynomial equation $p(x)$ with exactly the same roots. The method requires that the ratio $p(x) / f(x)$ be analytic in the interval under study. The concept shown here is that the correlation between the roots of a transcendental function and the Fourier series coefficients of its inverse can be expressed with a polynomial and proven with Cauchy's integral theorem.

The advantage of the current method is the possibility of solving for several roots in one shot and is most evident when dealing with an implicit problem as shown in the previous example. In this case, an implicit problem was transformed into a simpler explicit one. The current method remains explicit when dealing with up to four roots. However, the implicitness of finding roots of higher-order polynomials is not really an issue as algorithms designed for this task are readily available or larger problems can be divided into smaller problems with four roots each by selecting appropriate intervals as illustrated in Section 3. In any case, the current method provides a relatively simple solution procedure for a group of problems encountered in many engineering applications. 


\section{Notation}

Bi: Biot number

$c_{k}$ : $k$ th coefficient of the polynomial $P(y)$

$f$ : Function of $x$

$F$ : Function of $y$

$G$ : Function of $\theta$

$G_{k}: k$ th Fourier series coefficient of function $G$

$h$ : Center of a circle in the complex plane

$N$ : Number of roots

$p$ : Polynomial, function of $x$

$P$ : Polynomial, function of $y$

$r$ : Positive integer

$R$ : Radius of a circle in the complex plane

$x$ : Independent variable

$y$ : Normalized variable

$z$ : Complex variable

$\theta$ : Angle in the complex plane.

\section{Conflict of Interests}

The authors declare that there is no conflict of interests regarding the publication of this paper.

\section{References}

[1] J. S. Phelps III and M. J. Funcheon, "Solutions of a transcendental equation basic to the study of composite media heat transfer," Journal of Computational and Applied Mathematics, vol. 10, no. 2, pp. 135-139, 1984.

[2] J. W. Stevens and R. Luck, "Explicit approximations for all eigenvalues of the 1-D transient heat conduction equations," Heat Transfer Engineering, vol. 20, no. 2, pp. 35-41, 1999.

[3] A. Haji-Sheikh and J. V. Beck, "An efficient method of computing eigenvalues in heat conduction," Numerical Heat Transfer Part B: Fundamentals, vol. 38, no. 2, pp. 133-156, 2000.

[4] F. de Monte, "Multi-layer transient heat conduction using transition time scales," International Journal of Thermal Sciences, vol. 45, no. 9, pp. 882-892, 2006.

[5] S. Ruan and J. Wei, "On the zeros of transcendental functions with applications to stability of delay differential equations with two delays," Dynamics of Continuous, Discrete and Impulsive Systems, Series A: Mathematical Analysis, vol. 10, no. 6, pp. 863874, 2003.

[6] N. P. Aleshin, V. S. Kamenskii, and L. I. Mogil'ner, "Solution of a transcendental equation encountered in diffraction problems," Journal of Applied Mathematics and Mechanics, vol. 47, no. 1, pp. 139-141, 1983.

[7] C. M. Ruggiero, "Solution of transcendental and algebraic equations with applications to wave propagation in elastic plates," NASA STI/Recon Technical Report 81, 1981.

[8] G. Pickett and Y. Millev, "On the analytic inversion of functions, solution of transcendental equations and infinite selfmappings," Journal of Physics A: Mathematical and General, vol. 35, no. 20, pp. 4485-4494, 2002.

[9] S. C. Chapra and R. P. Canale, Numerical Methods for Engineers, McGraw-Hill, New York, NY, USA, 3rd edition, 1998.

[10] R. A. Leathers and N. J. McCormick, "Closed-form solutions for transcendental equations of heat transfer," Journal of Heat Transfer, vol. 118, no. 4, pp. 970-973, 1996.
[11] N. I. Muskhelishvili, Singular Integral Equations, Noordhoff, Groningen, The Netherlands, 1953.

[12] E. E. Burniston and C. E. Siewert, "The use of Riemann problems in solving a class of transcendental equations," Mathematical Proceedings of the Cambridge Philosophical Society, vol. 73, no. 1, pp. 111-118, 1973.

[13] R. Luck and J. W. Stevens, "Explicit solutions for transcendental equations," SIAM Review, vol. 44, no. 2, pp. 227-233, 2002.

[14] D. Jackson, "Non-essential singularities of functions of several complex variables," The Annals of Mathematics, vol. 17, no. 4, pp. 172-179, 1916.

[15] D. Jackson, "Roots and singular points of semi-analytic functions," The Annals of Mathematics, vol. 19, no. 2, pp. 142-151, 1917.

[16] E. Dehn, Algebraic Equations: An Introduction to the Theories of Lagrange and Galois, Columbia University Press, New York, NY, USA, 1930.

[17] J. M. McNamee, "A bibliography on roots of polynomials," Journal of Computational and Applied Mathematics, vol. 47, no. 3, pp. 391-394, 1993.

[18] J. M. McNamee, "A supplementary bibliography on roots of polynomials," Journal of Computational and Applied Mathematics, vol. 78, no. 1, p. 1, 1997.

[19] J. M. McNamee, "An updated supplementary bibliography on roots of polynomials," Journal of Computational and Applied Mathematics, vol. 110, no. 2, pp. 305-306, 1999.

[20] J. M. McNamee, Numerical Methods for Roots of Polynomials, Part 1, Studies in Computational Mathematics, vol. 14, Elsevier Science, Cambridge, Mass, USA, 2007.

[21] J. M. McNamee and V. Pan, Numerical Methods for Roots of Polynomials, Part II, vol. 16 of Studies in Computational Mathematics, Elsevier Science, Cambridge, Mass, USA, 2013.

[22] G. H. Golub and C. F. Van Loan, Matrix Computations, Johns Hopkins University Press, Baltimore, Md, USA, 3rd edition, 1996.

[23] Y. A. Cengel, Heat Transfer: A Practical Approach, McGraw-Hill, New York, NY, USA, 3rd edition, 2005.

[24] M. Abramowitz and I. A. Stegun, Eds., Handbook of Mathematical Functions with Formulas, Graphs, and Mathematical Tables, Dover Publications, New York, NY, USA, 9th edition, 1972.

[25] H. Press, S. A. Teukolsky, W. T. Vetterling, and B. R. Flannery, Numerical Recipes in Fortran 77: The Art of Scientific Computing, Cambridge University Press, 1992.

[26] E. W. Weisstein, CRC Concise Encyclopedia of Mathematics, CRC Press, Boca Raton, Fla, USA, 2nd edition, 2002. 


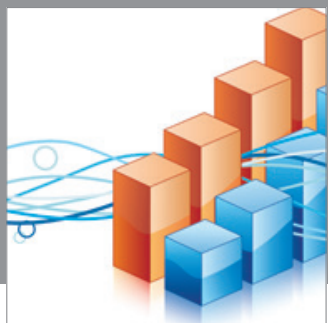

Advances in

Operations Research

mansans

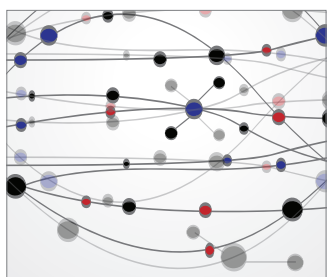

The Scientific World Journal
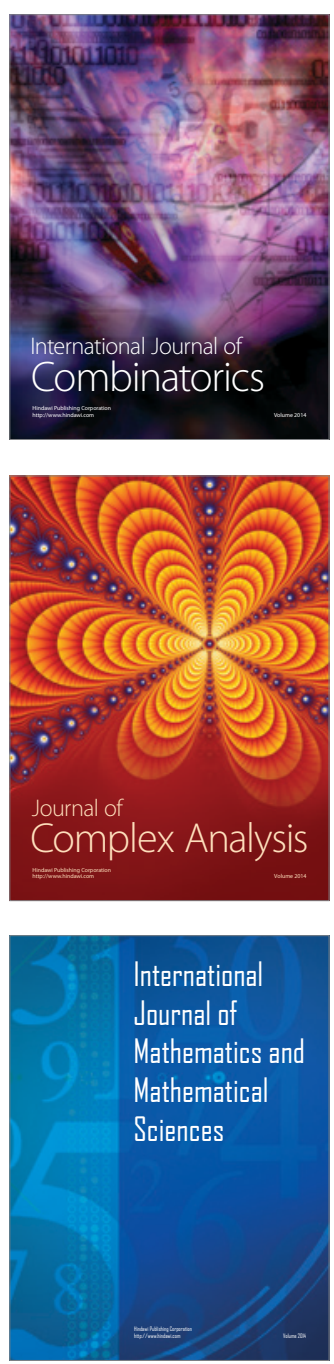
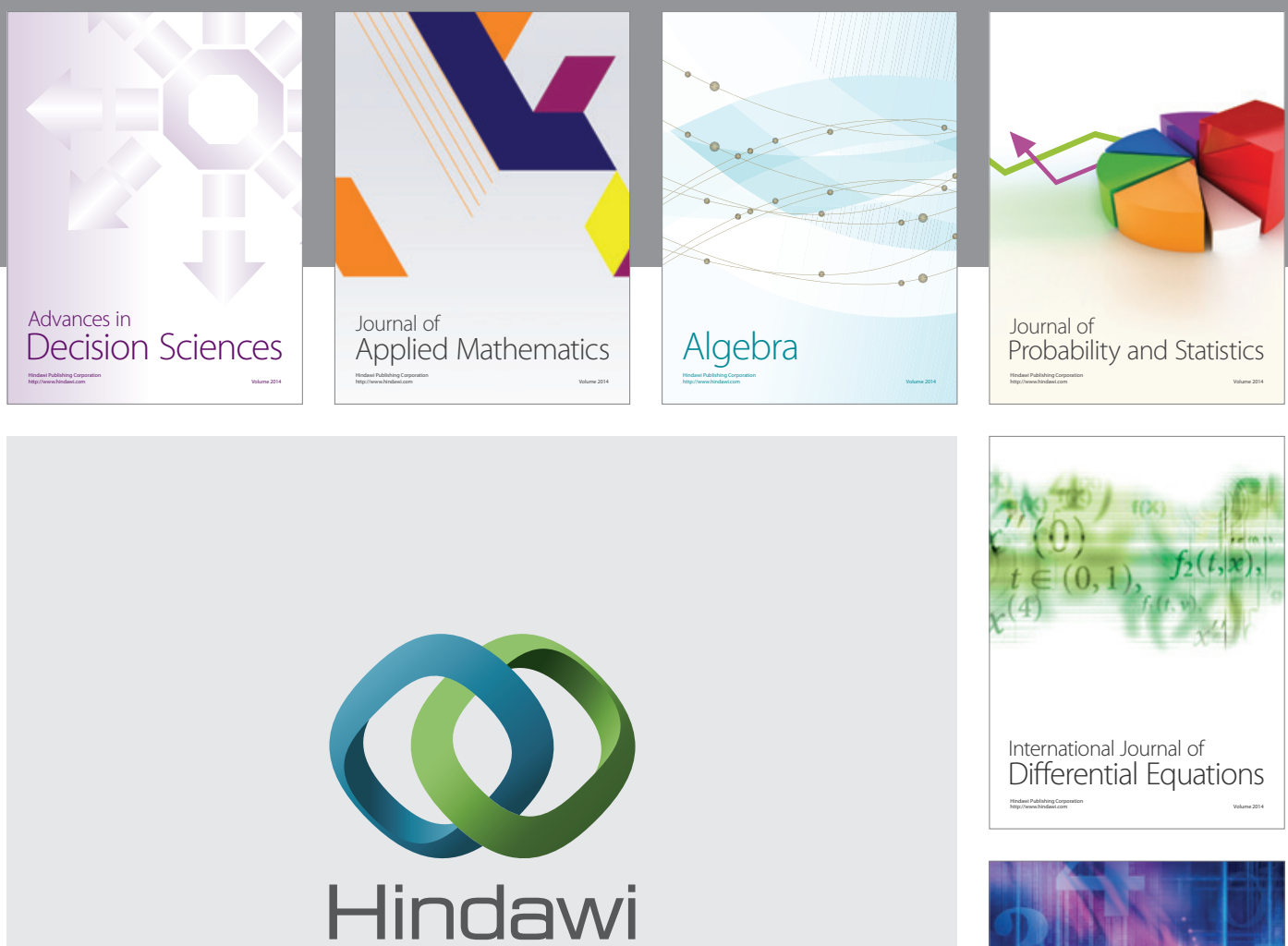

Submit your manuscripts at http://www.hindawi.com
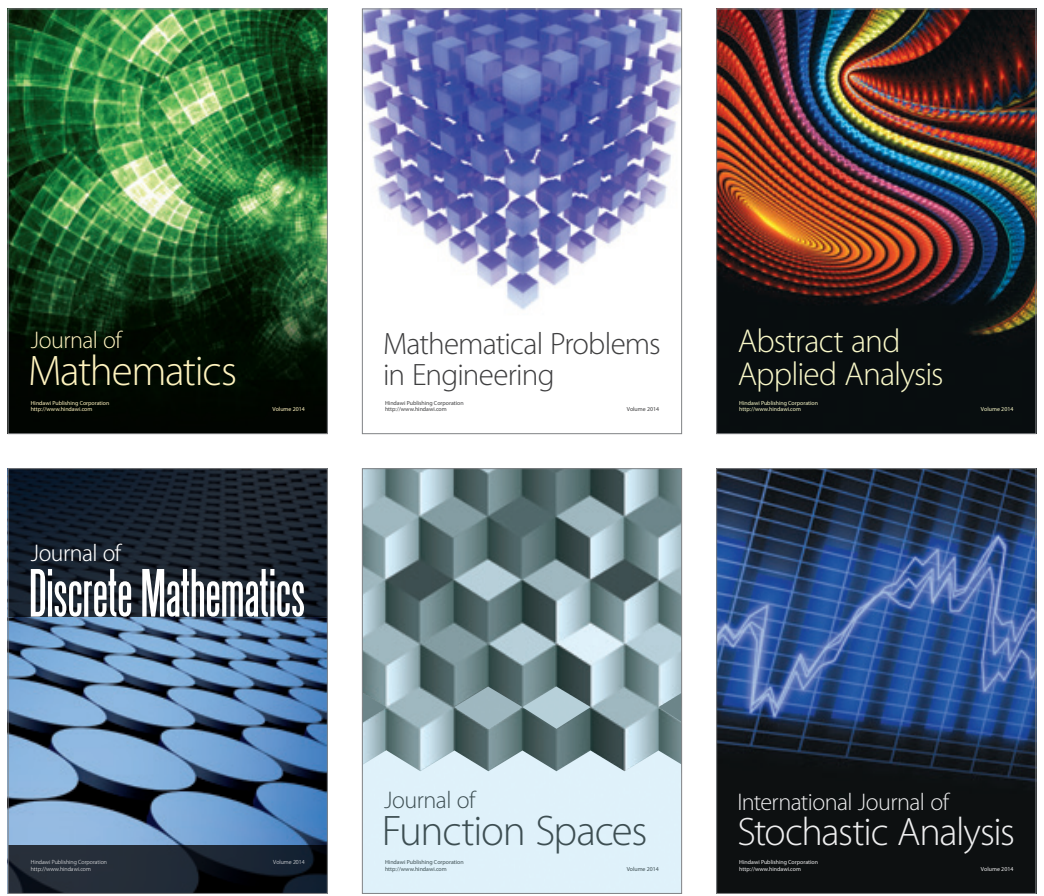

Journal of

Function Spaces

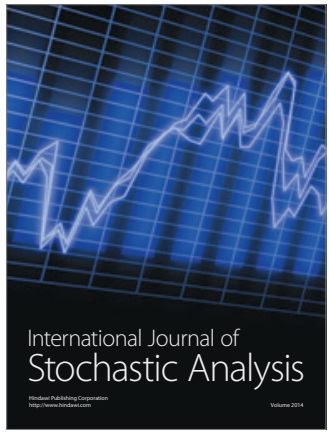

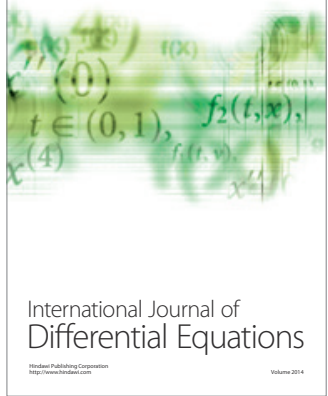
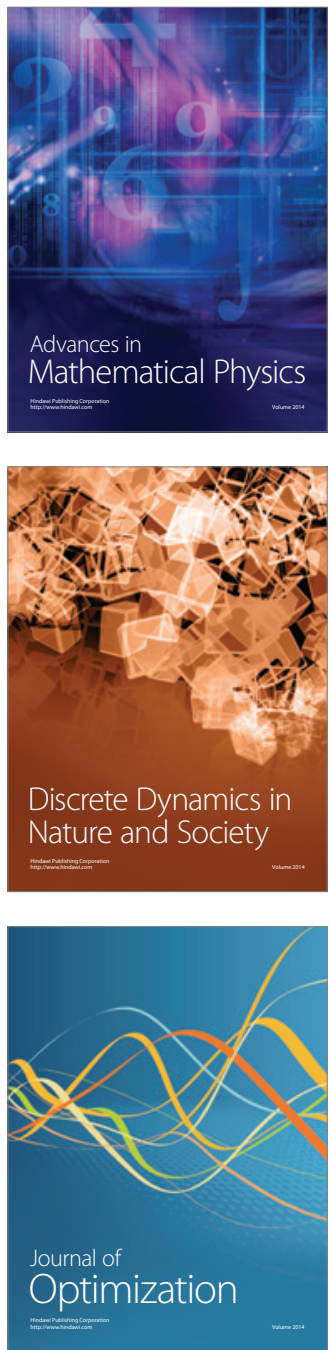